\title{
The Condemned Sinus: Natural Disease or Surgical Sequela?
}

\author{
Edward D. McCoul, MD, MPH, FACS ${ }^{1,2,3}$ \\ ${ }^{1}$ Department of Otorhinolaryngology, Ochsner Clinic Foundation, New Orleans, LA ${ }^{2}$ The University of Queensland School of Medicine, \\ Ochsner Clinical School, New Orleans, LA ${ }^{3}$ Department of Otolaryngology, Tulane University School of Medicine, New Orleans, LA
}

Background: Unilateral, mucopurulent drainage from an isolated paranasal sinus may be encountered in patients with a history of surgery for the treatment of chronic rhinosinusitis (CRS). Static mucus is visualized on nasal endoscopy within the sinus lumen but without significant disease in the adjacent sinuses. The reasons for this phenomenon are unknown although an iatrogenic cause is proposed.

Methods: A case series was prospectively compiled from consecutive patients presenting for evaluation of CRS at a tertiary rhinology practice during a 16-month period. Computerized tomography and nasal endoscopy were performed, and endoscopically directed aerobic and anaerobic bacterial cultures were obtained. Osteitis scores were recorded for diseased and nondiseased sides.

Results: Twenty-three of 113 patients (20.4\%) had evidence of chronic unilateral drainage from either a maxillary (21) or sphenoid (2) sinus. Mean osteitis scores were higher for the diseased side $(P<0.01)$. A nonendoscopic transantral approach was reported in $57.1 \%$ of cases with chronic maxillary disease, with $52.2 \%$ occurring more than 10 years earlier. The most common bacterial isolate was Pseudomonas aeruginosa (6 cases, $26.1 \%$ ), followed by methicillin-resistant Staphylococcus aureus (5 cases, $21.7 \%)$. Six cases (26.1\%) were polymicrobial, and $6(26.1 \%)$ were culture-negative. Tobacco use was reported in 8 (34.8\%) cases, and chronic obstructive pulmonary disease was present in $6(26.1 \%)$ cases.

Conclusion: The condemned sinus is a distinct entity that may represent a sequela of previous non-mucosal-sparing surgery. An association with hyperostosis is observed. Mucopurulent drainage is characterized by polymicrobial infection comparable to that found in diffuse CRS.

Keywords: Caldwell-Luc procedure, endoscopy, iatrogenic, osteitis, paranasal sinuses, sinusitis

Address correspondence to Edward D. McCoul, MD, MPH, FACS, Department of Otorhinolaryngology, Ochsner Clinic Foundation, 1514 Jefferson Hwy., New Orleans, LA 70121. Tel: (504) 842-4080. E-mail: edward.mccoul@ochsner.org

\section{INTRODUCTION}

Endoscopic sinus surgery (ESS) has undergone numerous refinements since its introduction in the 1980s. One of the most notable refinements has been a shift from surgery directed at exenterating or "scraping" the sinus cavity in favor of mucosal-sparing surgical techniques. This evolution has occurred in tandem with new discoveries into the function of the normal and diseased sinonasal tract. ${ }^{1}$ Surgical procedures from previous decades were focused on the goal of removing diseased mucosa from within the sinus lumen. ${ }^{2}$ Even after introduction of the endoscope, procedures such as ethmoidectomy and sphenoidotomy remained fundamentally similar to the original description of the Caldwell-Luc procedure that prescribed the removal of all maxillary sinus mucosa. ${ }^{3}$ Only gradually in the intervening decades has a shift toward mucosal preservation become a part of surgical practice. Today, avoidance of unnecessary mucosal disruption during ESS remains appropriate in the majority of cases, as mucosal inflammation is now understood to be a potentially reversible process. ${ }^{4}$

The mucosa of the paranasal sinuses is highly specialized, with known functions including mechanical clearance of toxins and foreign matter, nitric oxide production, and cellular and humoral adaptive immune responses. ${ }^{5}$ In the historic procedures described above, postoperative dysfunction as a result of injurious mucosal handling or wholesale mucosal stripping may be considered likely. However, little is known about the natural history of such mucosal damage in humans. In clinical practice, one or more sinuses may exhibit the signs of a condemned sinus, characterized by impaired mucociliary clearance with mucus stasis and chronic localized bacterial infection. This condition is potentially distinct from well-recognized forms of isolated or unilateral chronic sinusitis, such as mycetoma, odontogenic sinusitis, or antrochoanal polyp, that typically occur in 
patients without prior surgery. The purpose of this descriptive study was to identify defining characteristics in patients who manifested an isolated dysfunctional sinus in the setting of prior sinus surgery.

\section{METHODS}

A case series with planned data collection was prospectively compiled from all new patients presenting to a tertiary rhinology practice with a diagnosis of chronic rhinosinusitis (CRS) between January 2015 and April 2016. Diagnosis of CRS was made according to guidelines from the American Academy of Otolaryngology-Head and Neck Surgery and the European Position Paper on Rhinosinusitis and Nasal Polyps. ${ }^{6,7}$ All patients had computerized tomography (CT) of the paranasal sinuses and baseline nasal endoscopy. $\mathrm{Pa}-$ tients with evidence of prior sinus surgery were identified based on CT or endoscopic findings. Among those with prior surgery, patients were included who had a complaint of chronic, persistent postnasal drainage with a confirmatory finding of unilateral mucopurulent exudate on nasal endoscopy. Patients $<18$ years were excluded, as were patients without objective evidence of prior sinus surgery. Surgical records were reviewed when available. In all cases, a sinus swab for culture of aerobic and anaerobic bacteria was obtained under endoscopic visualization. Recorded data included demographics, the year and type of prior sinus surgery, prior surgical indication, and current sinus culture results. This study was approved by the Institutional Review Board of the Ochsner Clinic Foundation (protocol \#2015.170.C).
Radiographic changes of the bone of the sinus wall were assessed using a modification of the Global Osteitis Scoring Scale as previously described and validated elsewhere. ${ }^{8}$ Scoring was performed only for the maxillary or sphenoid sinuses, using a 5-point scale based on the extent of hyperostosis and the thickness of the bone. Rather than calculating a global total for all sinuses, only the score for the relevant sinus was evaluated. Diseased and nondiseased sides were compared using the Kruskal-Wallis test.

\section{RESULTS}

A total of 426 patients with CRS were evaluated during the study period, of which 113 had a history of prior sinus surgery. Twenty-three patients had a chronically draining sinus, yielding a prevalence of $20.4 \%$ among patients with a history of prior sinus surgery and of $5.4 \%$ among the screened population.

The mean (SD) age was 60.4 (12.3) years, and $69.6 \%$ of the patients were female. Twenty patients $(87.0 \%)$ were Caucasian; 3 (13.0\%) were African American. Twenty-one cases $(91.3 \%)$ involved the maxillary sinus, and 2 cases $(8.7 \%)$ involved the sphenoid sinus. Comorbid conditions included asthma in 2 patients $(8.7 \%)$ and chronic obstructive pulmonary disease in 6 patients $(26.1 \%)$. Eight patients $(34.8 \%)$ reported tobacco use. CT imaging in all patients revealed diffuse partial opacification of a patent maxillary or sphenoid sinus, with very minimal or absent disease in the remaining sinuses (Figure 1). The mean (SD) osteitis score for the condemned sinus was 2.7 (1.1) compared to $1.2(0.4)$ on the contralateral side $(P<0.01)$. Typical endoscopic findings

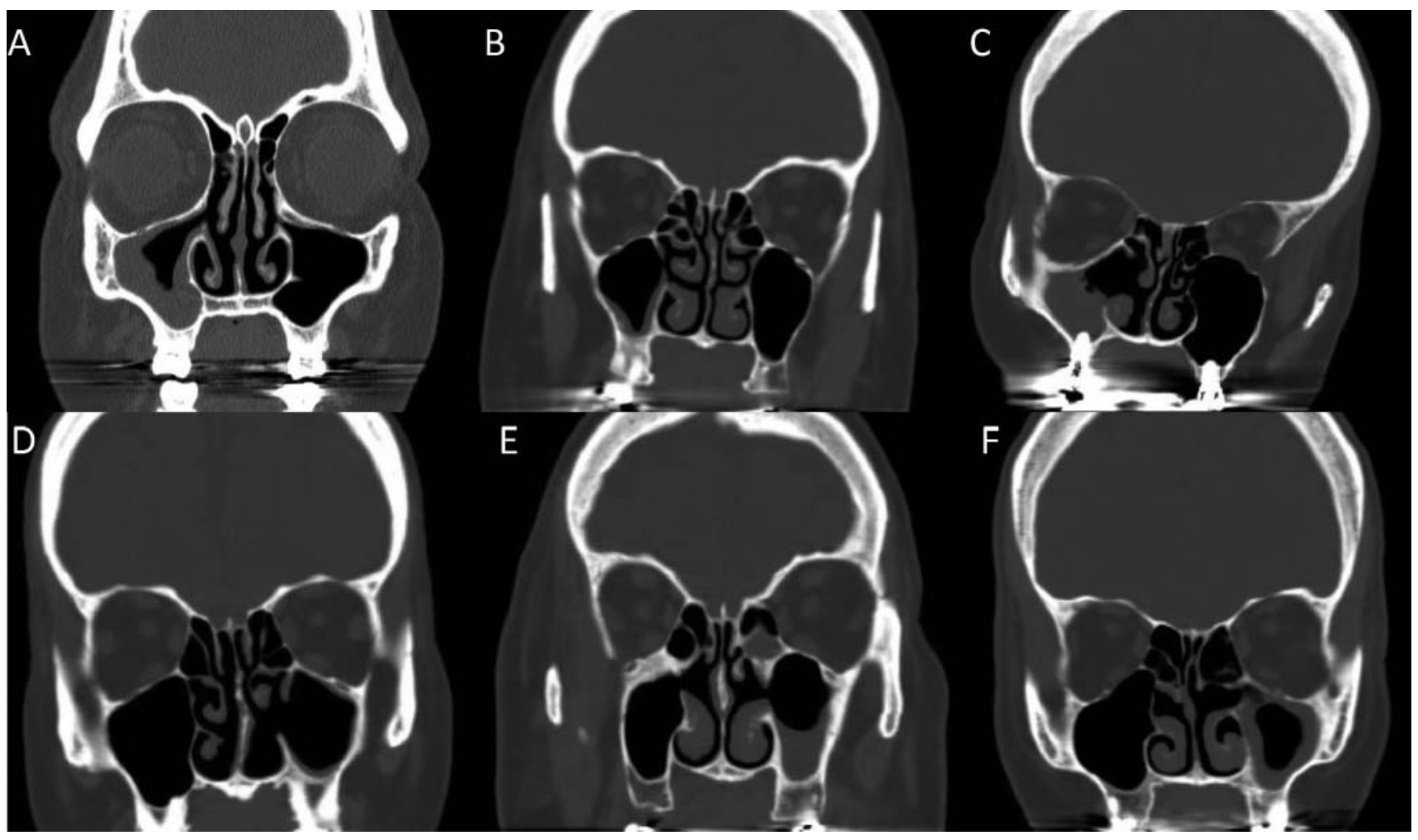

Figure 1. Computerized tomography shows the appearance of a chronically draining condemned sinus in 6 patients. In the first set of 3 cases (A-C), the patients' right maxillary sinus is dysfunctional; in the second set of 3 cases (D-F) the patients' left maxillary sinus is dysfunctional. 


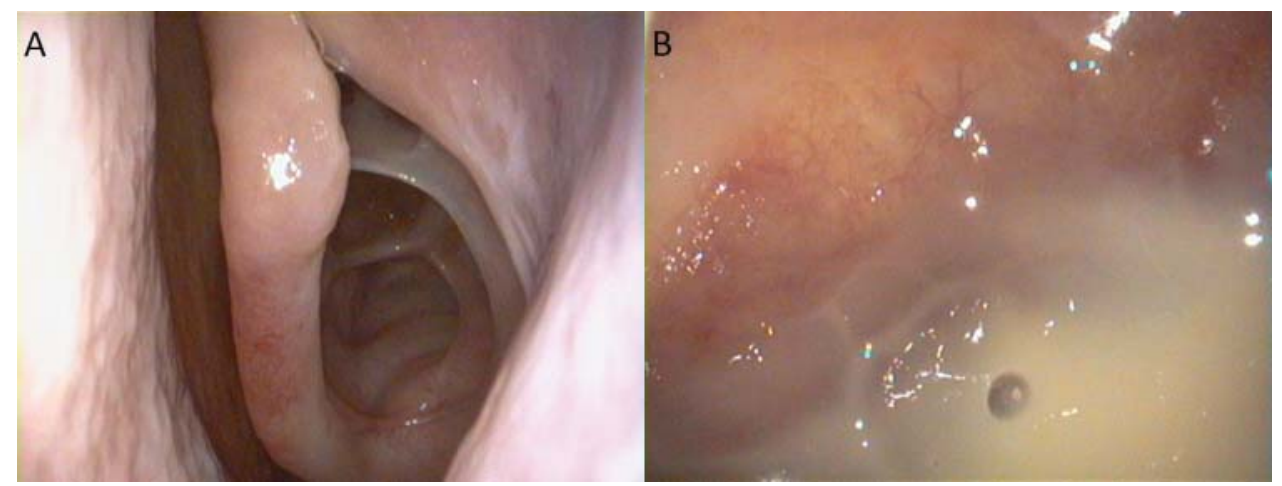

Figure 2. Endoscopic appearance of the left ethmoid (A) and left maxillary antrum (B) in a patient with a condemned maxillary sinus. Note the static puddle of purulence in the antrum (visible with the scope retroflexed into the lumen) despite a normal-appearing postoperative ethmoidectomy.

were patent sinuses that were well healed from prior surgery, with a collection of mucopurulent exudate within the dependent portion of the sinus lumen (Figure 2).

As stated, all patients had a history of prior surgery. Of the patients whose surgery involved the maxillary sinus, 12 (57.1\%) had evidence of transantral maxillary surgery via a Caldwell-Luc approach. Twelve patients (52.2\%) had had an initial sinus surgery performed prior to the year 2005, with a median in 2003 (range, 1985-2014). Among the 11 patients who exclusively had ESS, 7 (63.6\%) surgeries had been performed during the previous 10 years. The stated indications for prior surgery were fungal disease in 7 cases $(30.4 \%)$, polyposis in 6 cases $(26.1 \%)$, inverted papilloma in 2 cases $(8.7 \%)$, and odontogenic keratocyst in 1 case $(4.3 \%)$, with the remainder of indications unclear.

The 2 patients with isolated sphenoid sinusitis likewise demonstrated isolated partial opacification of that sinus with otherwise clear sinuses on CT (Figure 3). Endoscopy revealed mucopurulent drainage from a patent sphenoid sinusotomy (Figure 4).

Cultures of mucopurulent sinus exudate were positive in 17 cases $(73.9 \%)$. The most common isolate was Pseudomonas aeruginosa (6 cases, $26.1 \%$ ), followed by methicillinresistant Staphylococcus aureus (5 cases, 21.7\%). Six cases
(26.1\%) were polymicrobial. Bacterial isolates are summarized in the Table.

\section{DISCUSSION}

ESS has been shown to be beneficial in treating CRS, with putative benefits from reestablishing sinus ventilation, evacuating purulent material, debulking polyps, and restoring normal mucociliary clearance. Normal mucus clearance relies upon the principles of Messerklinger and others who identified the stereotypical pattern of mucociliary flow from the sinus lumen to the natural ostium. ${ }^{9}$ This concept underlies the rationale for functional ESS, in which natural ostia are identified and surgically enlarged to enhance the inherent sinus function. This approach is in contrast to traditional sinus surgery in which an opening was created at the most easily accessible or gravity-dependent location, often through a solid bony partition. Examples include frontal sinus trephination and inferior meatal antrostomy. ${ }^{10-12}$ This traditional rationale likened the goal of sinus surgery to the drainage of an abscess, on the principle that merely evacuating sinus contents would be sufficient to restore normal function. While these options may be viable for treating acute sinusitis, their effectiveness in treating appropriately diagnosed CRS has never been established.

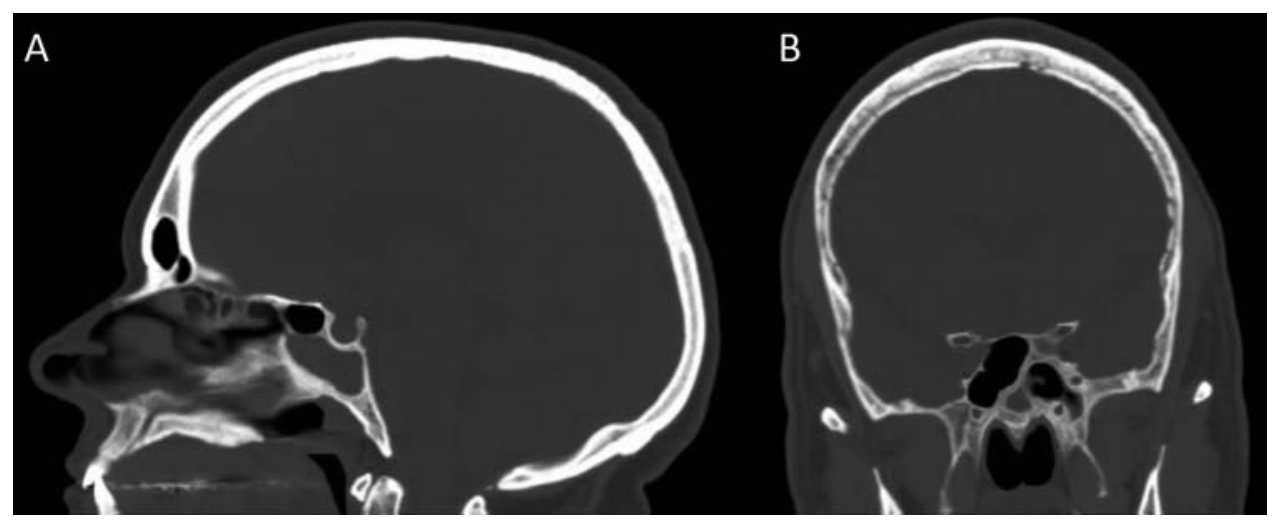

Figure 3. Computerized tomography shows the appearance of a chronically draining condemned sphenoid sinus in sagittal (A) and coronal (B) views. 


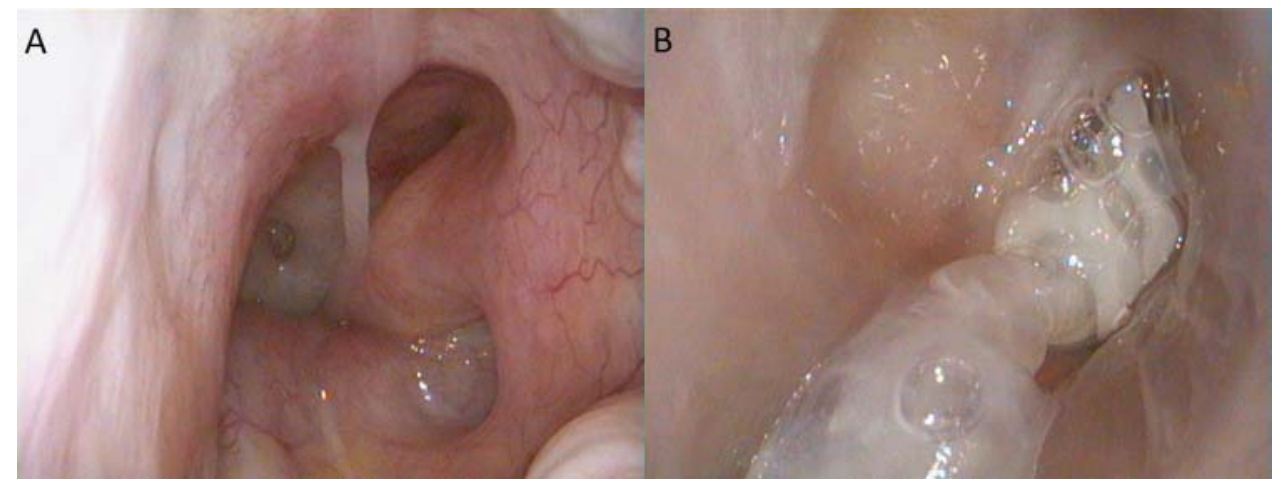

Figure 4. Endoscopic appearance of the left choana (A) and sphenoid ostium (B) in a patient with a condemned sphenoid sinus. A. A trickle of mucus is visible descending from the sphenoethmoidal recess. B. Closer examination with a 30-degree endoscope shows thick mucopurulent drainage emanating from the previously operated sphenoid ostium.

In addition to the site of the sinusotomy, traditional sinus surgery differs from ESS in the surgeon's willingness to sacrifice mucosa in addressing the disease process. For many years, the mainstay of treating maxillary sinusitis was the Caldwell-Luc procedure that entails a sublabial transantral approach adjacent to the canine fossa. Successful completion of this procedure was intended to include not only access to the sinus but also radical removal of mucosa that was assumed to be irreversibly diseased. ${ }^{13,14}$ With the removal of the native mucosa, and presumably the periosteum, the sinus would remain nonfunctional until new mucosa was generated. ${ }^{15}$ The natural history for this regeneration has not been defined; furthermore, the mucosa that regenerates may be deficient or dysfunctional in one or more of its intrinsic functions, potentially giving rise to a condemned sinus as described in this study.

Table. Cultured Isolates Obtained From Endoscopically Directed Swabs of Chronically Draining Sinuses $(n=23)$

\begin{tabular}{lcc}
\hline Isolate & $\begin{array}{c}\text { Number } \\
\text { of Cases }\end{array}$ & $\begin{array}{c}\text { Frequency, } \\
\%\end{array}$ \\
\hline Pseudomonas aeruginosa & 6 & 26.1 \\
$\begin{array}{l}\text { Staphylococcus aureus, } \\
\text { methicillin-resistant }\end{array}$ & 5 & 21.7 \\
Escherichia coli & 2 & \\
Enterobacter cloacae & 2 & 8.7 \\
Prevotella sp. & 2 & 8.7 \\
Staphylococcus aureus, & 1 & 8.7 \\
$\quad$ methicillin-sensitive & 1 & 4.3 \\
Klebsiella pneumoniae & 1 & 4.3 \\
Serratia marcescens & 1 & 4.3 \\
Citrobacter sp. & 1 & 4.3 \\
Propionibacterium sp. & 1 & 4.3 \\
Fusarium sp. & 6 & 4.3 \\
No growth & & 26.1
\end{tabular}

Note: Percentages total greater than $100 \%$ because of polymicrobial growth in 6 cases.
Despite the promulgation of ESS since the 1980s, it is conceivable that some surgeons adhere to the notion that exenteration of mucosa, rather than its preservation, is a valid component of surgical treatment. Even more probable is that unintentional stripping of mucosa by otherwise wellintentioned surgeons occurs as the result of inadequate instrumentation or technical infacility. In either scenario, a functional sinus may be converted to a condemned sinus as a sequela of surgical treatment.

Previous study has found an association between the extent of paranasal sinus hyperostosis and the number of previous sinus surgeries. ${ }^{8}$ Additionally, osteitic changes were most commonly found in the maxillary sinus and were correlated with the extent of mucosal disease on CT imaging. This relationship is reflected in the present study in which hyperostosis was significantly associated with the condemned sinus. It is unclear whether hyperostosis is a consequence of surgical intervention vs a manifestation of the underlying disease process, and further study is warranted.

The bacteria recovered from culturing the chronic drainage from a condemned sinus are consistent with the flora typically found in exacerbations of $\mathrm{CRS}^{16}$ : Staphylococcus, Enterobacter, Prevotella, and other mixed flora. In contrast, predominant organisms in acute sinusitis are most often Streptococcus pneumoniae, Haemophilus influenzae, and Moraxella catarrhalis. ${ }^{17}$ This bacteriology supports the cases in this series being chronic rather than acute processes. Cases with negative culture may be attributed to biofilmmediated disease in which insufficient bacteria exist in planktonic form to permit culture by standard methods. ${ }^{18}$

The condemned sinus seems to occur preferentially in the maxillary sinus, with a small minority in the sphenoid and none in the frontal. This occurrence reflects the typical incidence of both sinus disease and the surgery that is used to treat it, with the maxillary sinus being most common in both categories. A condemned sinus may be unlikely to occur in the frontal sinus because that sinus is the most difficult to access during surgery, and stripping the mucosa would be difficult using standard instrumentation.

In the present case series, we found that a majority of patients with a condemned sinus had undergone surgery more than 10 years previously. Although direct inference is not possible from time of surgery alone, surgery performed in 
this time frame could conceivably have been more likely to include deliberate stripping of mucosa by the surgeon. Among the patients with a condemned maxillary sinus, a majority had undergone a Caldwell-Luc procedure that also would increase the probability that mucosal exenteration had occurred during the surgery.

An interesting finding of the present series is that despite a stated surgical indication of either polyposis or fungal sinusitis in a majority of cases, none of the condemned sinuses had any physical sign of these processes at the most recent evaluation. This finding may support the assertion that the mucosa was exenterated along with the diseased tissue during the initial surgery, with subsequent regeneration of dysfunctional mucosa. Anecdotally, many of the patients in this study reported that the chronic drainage began months or years after the initial surgery performed to treat another indication. Longitudinal study of a large surgical cohort would be useful to further investigate this putative process, including the time to disease manifestation, extent of mucosal injury, and other contributing factors.

\section{CONCLUSION}

A chronically draining condemned sinus may occur as a sequela of prior sinus surgery in which trauma occurred to the native mucosa. This condition may be associated with prior Caldwell-Luc antrostomy and often occurs following surgery performed for another indication. The condemned sinus is associated with hyperostosis, and the chronic mucopurulent drainage is characterized by bacteria resembling those found in other forms of CRS. Clinicians should be aware of the potential consequences of deliberate or inadvertent disruption of the sinus mucosa during surgery, as well as radiographic findings that may suggest the presence of this condition.

\section{ACKNOWLEDGMENTS}

These findings were presented in part at the American Rhinologic Society Spring Meeting in San Diego, CA, on April 27-28, 2017. The author has no financial or proprietary interest in the subject matter of this article.

\section{REFERENCES}

1. Stammberger $\mathrm{H}$, Posawetz W. Functional endoscopic sinus surgery. Concept, indications and results of the Messerklinger technique. Eur Arch Otorhinolaryngol. 1990;247(2):63-76.

2. Blitzer A, Lawson W. The Caldwell-Luc procedure in 1991. Otolaryngol Head Neck Surg. 1991 Nov;105(5):717-722.
3. Tange RA. Some historical aspects of the surgical treatment of the infected maxillary sinus. Rhinology. 1991 Jun;29(2):155-162.

4. Lund V. The evolution of surgery on the maxillary sinus for chronic rhinosinusitis. Laryngoscope. 2002 Mar;112(3):415-419.

5. Beule AG. Physiology and pathophysiology of respiratory mucosa of the nose and the paranasal sinuses. GMS Curr Top Otorhinolaryngol Head Neck Surg. 2010;9:Doc07. doi: 10.3205/ cto000071.

6. Rosenfeld RM, Andes D, Bhattacharyya N, et al. Clinical practice guideline: adult sinusitis. Otolaryngol Head Neck Surg. 2007 Sep;137(3 Suppl):S1-S31.

7. Fokkens WJ, Lund VJ, Mullol J, et al. EPOS 2012: European position paper on rhinosinusitis and nasal polyps 2012. A summary for otorhinolaryngologists. Rhinology. 2012 Mar;50 (1):1-12. doi: 10.4193/Rhino50E2.

8. Georgalas C, Videler W, Freling N, Fokkens W. Global Osteitis Scoring Scale and chronic rhinosinusitis: a marker of revision surgery. Clin Otolaryngol. 2010 Dec;35(6):455-461. doi: 10.1111/ j.1749-4486.2010.02218.x.

9. Stammberger $\mathrm{H}$. Endoscopic endonasal surgery-concepts in treatment of recurring rhinosinusitis. Part II. Surgical technique. Otolaryngol Head Neck Surg. 1986 Feb;94(2):147-156.

10. Ruoppi $P$, Seppä J, Nuutinen J. Acute frontal sinusitis: etiological factors and treatment outcome. Acta Otolaryngol. 1993 Mar;113(2):201-205.

11. Lund VJ. The results of inferior and middle meatal antrostomy under endoscopic control. Acta Otorhinolaryngol Belg. 1993;47 (1):65-71.

12. Lund VJ. Inferior meatal antrostomy. Fundamental considerations of design and function. J Laryngol Otol Suppl. 1988;15:1-18.

13. Buiter CT. Nasal antrostomy. Rhinology. 1988 Mar;26(1):5-18.

14. Bernstein L. The Caldwell-Luc operation. Otolaryngol Clin North Am. 1971 Feb;4(1):69-77.

15. Gutman $M$, Houser S. latrogenic maxillary sinus recirculation and beyond. Ear Nose Throat J. 2003 Jan;82(1):61-63.

16. Brook I, Yocum P, Frazier EH. Bacteriology and beta-lactamase activity in acute and chronic maxillary sinusitis. Arch Otolaryngol Head Neck Surg. 1996 Apr;122(4):418-422; discussion 423.

17. Brook I. Bacteriology of chronic sinusitis and acute exacerbation of chronic sinusitis. Arch Otolaryngol Head Neck Surg. 2006 Oct;132(10):1099-1101.

18. Kilty SJ, Desrosiers MY. The role of bacterial biofilms and the pathophysiology of chronic rhinosinusitis. Curr Allergy Asthma Rep. 2008 May;8(3):227-233.

This article meets the Accreditation Council for Graduate Medical Education and the American Board of Medical Specialties Maintenance of Certification competencies for Patient Care, Medical Knowledge, and Practice-Based Learning and Improvement. 